\title{
Modelling of Short Term Interest Rate Based on Fractional Relaxation Equation
}

\author{
K. JAWORSKA \\ Institute of Mathematics and Cryptology \\ Military University of Technology, Kaliskiego 2, 00-908 Warszawa, Poland \\ In this paper, we try to model the dynamics of short term interest rate \\ using the fractional nonhomogeneous differential equation with stochastic \\ free term. This type of equation is similar to one which represents the vis- \\ coelastic behavior of certain materials from rheologic point of view. As a final \\ result we obtain the closed formula for prices of zero-coupon bonds. They \\ are analogous to those in Vasiček model, where instead of the exponential \\ functions we have the Mittag-Leffler ones.
}

PACS numbers: 89.65.Gh

\section{Motivation}

One of the most basic tasks of actuaries and financial analysts lies in computing present values of various cash flow streams. No matter how complicated the pattern of cash flow, this is straightforward in a world of certainty. In the real world the presence of stochastic interest rates complicates matters considerably, even for the simplest cash flow streams.

The interest rate market tells us how the value of money today is linked to its value in the future. As for share prices, foreign exchange or stocks indices, future values of interest rates are uncertain and therefore call for modelling by stochastic processes. In contrast to a share price, we do not expect an interest rate $r(t)$ to grow on average in an exponential way, but rather to fluctuate in a reasonable range around some fixed value.

It is well-known fact that there are many significant analogies between dynamics and stochastics of complex physical and economical systems. The methods and models, which describe physical phenomena, become very useful background in analysis of economical phenomena, cf. [1-3]. This likeness brought us to fractional relaxation equation.

\section{Introduction}

A basic interest bearing security is a discount or zero-coupon bond. It pays 1 PLN, say, at time of maturity $T$. The main question is: how much is the asset worth at time $T_{0}<T_{1}$ ? 
We write $B\left(T_{0}, T_{1}\right)$ for the price of a zero-coupon bond at time $T_{0} \leq T_{1}$. Clearly $B\left(T_{1}, T_{1}\right)=1$ PLN. Hence the price of a zero-coupon bond in contrast to a share price, depends on two dates. This matters a lot because bonds with different maturities are dependent: the 20-year and 10-year bond will behave in a very similar way on a short term basis, and therefore they will not be traded separately.

The classical model of interest rate term structure takes

$$
B\left(T_{0}, T_{1}\right)=\frac{B_{T_{0}}}{B_{T_{1}}},
$$

where $B_{t}$ is a deterministic accumulation process (saving account with floating interest rate). In fact, today, one does not know the future interest rate of saving account. This is why the recent models describe $B_{t}$ as a stochastic process on some probability space $(\Omega, \mathcal{M}, P)$ with filtration $\mathcal{F}_{t}$ and

$$
B\left(T_{0}, T_{1}\right)=\mathbb{E}_{Q}\left(\frac{B_{T_{0}}}{B_{T_{1}}} \mid \mathcal{F}_{T_{0}}\right) .
$$

$P$ is a real measure and $Q$ is some measure equivalent to $P$ (see [4] for probabilistic notation and [5] for basics of mathematical finance). Then one may take the formula (2) as weighted average of all possible values of random variable $\frac{B_{T_{0}}}{B_{T_{1}}}$.

Let us assume that $r(t)$ is $\mathcal{F}_{t}$-adapted process modelling interest rate of bank account (the so-called short term interest rate). Then a bond value is given as follows:

$$
B\left(T_{0}, T_{1}\right)=\mathbb{E}_{Q}\left[\exp \left(-\int_{T_{0}}^{T_{1}} r(s) \mathrm{d} s\right) \mid \mathcal{F}_{T_{0}}\right] \quad \forall T_{0} \in\left[0, T_{1}\right]
$$

and most often $r(t)$ is modelled as Itô process (diffusion process) with dynamics described by differential Itô equation

$$
\mathrm{d} r(t)=\mu(t, r(t)) \mathrm{d} t+\sigma(t, r(t)) \mathrm{d} W(t),
$$

where $W(t)$ is $Q$-Wiener process adapted to the filtration $\mathcal{F}_{t}$.

It is well known that we have two types of stochastic differential equations: Itô and Stratonowicz ones cf. [6]. The solution of Itô Eq. (4) is also a solution of Stratonowicz equation

$$
\mathrm{d} r(t)=\widetilde{\mu}(t, r(t)) \mathrm{d} t+\sigma(t, r(t)) \mathrm{d} W(t),
$$

with

$$
\widetilde{\mu}(t, r(t))=\mu(t, r(t))-\frac{1}{2} \sigma(t, r(t)) \frac{\partial \sigma(t, r)}{\partial r} .
$$

And successively the solution of (5) proves a solution of the equation, which is commonly termed as the Langevin equation

$$
\frac{\mathrm{d} r(t)}{\mathrm{d} t}=\widetilde{\mu}(t, r(t))+\sigma(t, r(t)) \xi(t),
$$

where $\xi(t)$ is generalized stochastic process idealized as so-called white noise.

Let us replace the classic differential operator in (7) by fractional differential operator cf. [7] 
$D_{0_{+}}^{\alpha} r(t)=\widetilde{\mu}(t, r(t))+\sigma(t, r(t)) \xi(t), \quad 0<\alpha \leq 1$.

The fractional derivative operators have allowed for the modelling of some special complex systems in nature with anomalous dynamics, such as the atmospheric diffusion of pollution, cellular diffusion processes, network traffic, signal transmission through strong magnetic fields, the effect of speculation on the profitability of stocks in financial markets, and any more. The point is whether the solution $r(t)$ of Eq. (7) will model the interest rate more precisely than the solution of the classical one?

\section{The Vasiček model}

Let us try to answer this question in case of the Vasiček model of interest rate. Standard model is the following:

$$
\mathrm{d} r(t)=(a-b r(t)) \mathrm{d} t+\sigma \mathrm{d} W(t), \quad a, b, \sigma=\text { const }>0,
$$

with initial condition $r(0)=r_{0}$.

In this case $\mu=\widetilde{\mu}$, so the Stratonowicz equation and Eq. (9) have the same solution

$$
r(t)=r_{0} \exp (-b t)+\frac{a}{b}(1-\exp (-b t))+\sigma \int_{0}^{t} \exp (-b(t-u)) \mathrm{d} W(u) .
$$

Let us note that the deterministic part of $r(t)$ tends to $a / b$, when $t \rightarrow \infty$.

The stochastic component has the expected value 0 and its variance tends to $\sigma^{2} / 2 b$.

\section{Fractional generalization of the Vasiček model}

We consider now the Langevin equation corresponding to Eq. (9):

$$
\frac{\mathrm{d} r(t)}{\mathrm{d} t}=(a-b r(t))+\sigma \xi(t) .
$$

Fractional generalized Langevin equation takes the following form:

$$
D_{0_{+}}^{\alpha} r(t)=(a-b r(t))+\sigma \xi(t), \quad 0<\alpha \leq 1 .
$$

Due to linearity, the solution $r(t)$ of Eq. (12) is the sum of two components:

- the general solution of deterministic equation

$$
D_{0_{+}}^{\alpha} r(t)+b r(t)=a
$$

and

- the particular solution of stochastic equation

$D_{0_{+}}^{\alpha} r(t)+b r(t)=\sigma \xi(t)$.

TheOREM 4.1. The general solution of deterministic equation

$$
D_{0_{+}}^{\alpha} r(t)+b r(t)=a, \quad t>0
$$

has the following form:

where

$$
r_{\operatorname{det}}(t)=C t^{\alpha-1} E_{\alpha, \alpha}\left(-b t^{\alpha}\right)+a t^{\alpha} E_{\alpha, \alpha+1}\left(-b t^{\alpha}\right), \quad C \in \mathbb{R},
$$




$$
E_{\alpha, \beta}(z):=\sum_{k=0}^{\infty} \frac{z^{k}}{\Gamma(\alpha k+\beta)}, \quad z, \beta \in \mathbb{C}, \quad \operatorname{Re} \alpha>0,
$$

is the Mittag-Leffler function.

The proof is based on Laplace transform cf. [7] cor. 5.1 and th. 5.4. Let us note that like $r(t)$ in classical Vasiček model, here $r_{\text {det }}(t)$ tends to $a / b$, when $t \rightarrow \infty$.

THEOREM 4.2. The particular solution of stochastic equation

$$
D_{0_{+}}^{\alpha} r(t)+b r(t)=\sigma \xi(t), \quad t>0
$$

has the following form:

$r_{\text {stoch }}(t)=\sigma t^{\alpha-1} E_{\alpha, \alpha}\left(-b t^{\alpha}\right) * \xi(t)$.

Proof. After the properties of Laplace transform cf. [8] we obtain

$$
\begin{aligned}
D_{0_{+}}^{\alpha} & r_{\text {stoch }}(t)=\mathcal{L}^{-1}\left[s^{\alpha} \mathcal{L}\left[r_{\text {stoch }}(t)\right](s)\right] \\
& =\mathcal{L}^{-1}\left[s^{\alpha} \mathcal{L}\left[\sigma t^{\alpha-1} E_{\alpha, \alpha}\left(-b t^{\alpha}\right) * \xi(t)\right](s)\right] \\
& =\mathcal{L}^{-1}\left[s^{\alpha} \sigma \mathcal{L}\left[t^{\alpha-1} E_{\alpha, \alpha}\left(-b t^{\alpha}\right)\right](s) \cdot \mathcal{L}[\xi(t)](s)\right] \\
& =\mathcal{L}^{-1}\left[s^{\alpha} \sigma \frac{1}{s^{\alpha}+b} \cdot \mathcal{L}[\xi(t)](s)\right]=\mathcal{L}^{-1}\left[\sigma\left(1-\frac{b}{s^{\alpha}+b}\right) \cdot \mathcal{L}[\xi(t)](s)\right] \\
& =\sigma \mathcal{L}^{-1}[\mathcal{L}[\xi(t)](s)]-\sigma b \mathcal{L}^{-1}\left[\frac{1}{s^{\alpha}+b} \cdot \mathcal{L}[\xi(t)](s)\right] \\
& =\sigma \xi(t)-b \sigma \mathcal{L}^{-1}\left[\mathcal{L}\left[t^{\alpha-1} E_{\alpha, \alpha}\left(-b t^{\alpha}\right)\right](s) \cdot \mathcal{L}[\xi(t)](s)\right] \\
& =\sigma \xi(t)-b \sigma t^{\alpha-1} E_{\alpha, \alpha}\left(-b t^{\alpha}\right) * \xi(t)=\sigma \xi(t)-b r_{\text {stoch }}(t) .
\end{aligned}
$$

REMARK 4.1. If $0.5<\alpha \leq 1$ then $r_{\text {stoch }}(t)$ can be expressed as a stochastic integral

$$
r_{\text {stoch }}(t)=\sigma \int_{0}^{t}(t-s)^{\alpha-1} E_{\alpha, \alpha}\left(-b(t-s)^{\alpha}\right) \mathrm{d} W(s) .
$$

\section{Zero-coupons bonds pricing}

For the pricing of zero-coupon bonds it is useful to introduce an auxiliary stochastic process $Y\left(T_{0}, t\right)$ depending on the parameter $T_{0}$. We put

$$
B\left(T_{0}, T_{1}\right)=\mathbb{E}_{Q}\left(\exp \left(-Y\left(T_{0}, T_{1}\right) \mid \mathcal{F}_{T_{0}}\right),\right.
$$

where

$$
Y^{\prime}\left(T_{0}, t\right)=r(t)
$$

and

$$
Y\left(T_{0}, T_{0}\right)=0 \text {. }
$$

We separate from $Y\left(T_{0}, T_{1}\right)$ deterministic and stochastic parts $Y\left(T_{0}, T_{1}\right)=Y_{\text {det }}\left(T_{0}, T_{1}\right)+Y_{\text {stoch }}\left(T_{0}, T_{1}\right)$, 


$$
\begin{aligned}
Y_{\text {det }} & \left(T_{0}, T_{1}\right)=\int_{T_{0}}^{T_{1}} r_{\text {det }}(t) \mathrm{d} t \\
& =\left.C t^{\alpha} E_{\alpha, \alpha+1}\left(-b t^{\alpha}\right)\right|_{T_{0}} ^{T_{1}}+\left.a t^{\alpha+1} E_{\alpha, \alpha+2}\left(-b t^{\alpha}\right)\right|_{T_{0}} ^{T_{1}}, \\
Y_{\text {stoch }} & \left(T_{0}, T_{1}\right)=\left.\sigma t^{\alpha} E_{\alpha, \alpha+1}\left(-b t^{\alpha}\right) * \xi(t)\right|_{T_{0}} ^{T_{1}} \\
& =\sigma \int_{0}^{T_{1}}\left(T_{1}-s\right)^{\alpha} E_{\alpha, \alpha+1}\left(-b\left(T_{1}-s\right)^{\alpha}\right) \mathrm{d} W(s) \\
& -\sigma \int_{0}^{T_{0}}\left(T_{0}-s\right)^{\alpha} E_{\alpha, \alpha+1}\left(-b\left(T_{0}-s\right)^{\alpha}\right) \mathrm{d} W(s) \\
& =\sigma \int_{0}^{T_{0}}\left[\left(T_{1}-s\right)^{\alpha} E_{\alpha, \alpha+1}\left(-b\left(T_{1}-s\right)^{\alpha}\right)\right. \\
& \left.-\left(T_{0}-s\right)^{\alpha} E_{\alpha, \alpha+1}\left(-b\left(T_{0}-s\right)^{\alpha}\right)\right] \mathrm{d} W(s) \\
& +\sigma \int_{T_{0}}^{T_{1}}\left(T_{1}-s\right)^{\alpha} E_{\alpha, \alpha+1}\left(-b\left(T_{1}-s\right)^{\alpha}\right) \mathrm{d} W(s) \\
& =Y_{\text {stoch } 1}\left(T_{0}, T_{1}\right)+Y_{\text {stoch } 2}\left(T_{0}, T_{1}\right) .
\end{aligned}
$$

Hence we get decomposition

$$
Y_{\text {stoch }}\left(T_{0}, T_{1}\right)=Y_{\text {stoch } 1}\left(T_{0}, T_{1}\right)+Y_{\text {stoch2 }}\left(T_{0}, T_{1}\right),
$$

where $Y_{\text {stoch } 1}\left(T_{0}, T_{1}\right)$ is $\mathcal{F}_{T_{0}}$ measurable, $Y_{\text {stoch2 }}\left(T_{0}, T_{1}\right)$ is $\mathcal{F}_{T_{1}}$ measurable, independent of $\mathcal{F}_{T_{0}}$ and has normal distribution $N\left(0, \Sigma^{2}\right)$,

$$
\Sigma^{2}=\sigma^{2} \int_{T_{0}}^{T_{1}}\left(T_{1}-s\right)^{2 \alpha} E_{\alpha, \alpha+1}^{2}\left(-b\left(T_{1}-s\right)^{\alpha}\right) \mathrm{d} s .
$$

THEOREM 5.1.

$$
B\left(T_{0}, T_{1}\right)=\exp \left(-Y_{\operatorname{det}}\left(T_{0}, T_{1}\right)-Y_{\text {stoch1 }}\left(T_{0}, T_{1}\right)+\frac{\Sigma^{2}}{2}\right) .
$$

Proof. able.

We apply the formula for the expected value of the log-normal random vari-

$$
\begin{aligned}
& B\left(T_{0}, T_{1}\right)=\mathbb{E}_{Q}\left(\exp \left(-Y_{\operatorname{det}}\left(T_{0}, T_{1}\right)-Y_{\text {stoch } 1}\left(T_{0}, T_{1}\right)-Y_{\text {stoch2 }}\left(T_{0}, T_{1}\right)\right) \mid \mathcal{F}_{T_{0}}\right) \\
& \quad=\exp \left(-Y_{\operatorname{det}}\left(T_{0}, T_{1}\right)\right) \exp \left(-Y_{\text {stoch } 1}\left(T_{0}, T_{1}\right)\right) \mathbb{E}_{Q}\left(\exp \left(-Y_{\text {stoch2 }}\left(T_{0}, T_{1}\right)\right)\right) \\
& \quad=\exp \left(-Y_{\operatorname{det}}\left(T_{0}, T_{1}\right)\right) \exp \left(-Y_{\text {stoch1 }}\left(T_{0}, T_{1}\right)\right) \exp \left(\frac{\Sigma^{2}}{2}\right)
\end{aligned}
$$

\section{Conclusions}

The fractional differential operators are powerful tools used successfully in various areas of science and engineering. One of the key of these applications is that under a certain limit the fractional theory includes the classical. As it 
is expected the dynamics of the fractional calculus systems is different from the classical one, but the classical dynamics is recovered as a particular case.

In this paper we generalized the classical Vasiček model and obtained a new term structure model, which emphasizes the impact of the fluctuations of historical interest rates on the actual prices of bonds with different maturities. The classical results are reobtained when the degree of the fractional operator $\alpha=1$.

The solution of fractional differential equation is represented in the integral form. However it is possible to make effective numerical computations which give fair results satisfying financial analysts.

\section{References}

[1] R.N. Mantegna, H.E. Stanley, An Introduction to Econophysics. Correlations and Complexity in Finance, Cambridge University Press, Cambridge 2000, p. 158.

[2] M. Kozłowska, R. Kutner, Acta Phys. Pol. B 37, 3027 (2006).

[3] M. Kozłowska, A. Kasprzak, R. Kutner, Int. J. Mod. Phys. C 19, 1 (2008).

[4] P. Billingsley, Probability and Measure, Wiley, New York 1979, p. 600.

[5] D. Heath, in: Introduction to Mathematical Finance, Eds. D. Heath, G. Swindle, Proc. Symp. Appl. Math., Vol. 57, AMS, Providence 1999, p. 180.

[6] K. Sobczyk, Stochastic Differential Equations: with Applications to Physics and Engineering, Kluwer Academic Publishers, Dordrecht 1991, p. 416.

[7] A.A. Kilbas, H.M. Srivastava, J.J. Trujillo, Theory and Applications of Fractional Differential Equations, Elsevier B.V., Amsterdam 2006, p. 540.

[8] Z. Szmydt, B. Ziemian, The Mellin Transformation and Fuchsian Type Partial Differential Equations, Kluwer Academic Publishers, Dordrecht 1992, p. 240. 